\section{Clinical Stevens-Johnson syndrome and rufinamide: A clinical case}

\section{To the Editor}

Toxic epidermal necrolysis (TEN) and Stevens-Johnson syndrome (SJS) are rare but extremely serious mucocutaneous reactions characterised by systemic symptoms and extensive epithelial sloughing; epidermal detachment of less than $10 \%$ body surface area is considered as SJS. 1,2,4 Drugs are the commonest causes, including antiepileptics and antibiotics. ${ }^{1,4}$ Infections, like Mycoplasma pneumonia, are the second commonest cause. Prompt diagnosis is essential; early hospitalisation of patients is of outmost importance ${ }^{2}$ and any suspected causative drug must be stopped immediately.

Rufinamide is an antiepileptic drug, chemically not related with other anticonvulsants. It was approved in 2007 as an adjuvant drug in patients older than four years of age with Lennox-Gastaut syndrome. We found no previous report of SJS related to rufinamide.

We report the case of a 13-year-old girl, with the diagnosis of West syndrome and global psychomotor delay. She was treated with valproic acid, clonazepam and levetiracetam; as seizures were uncontrolled, rufinamide was added to the usual medication (600 mg, twice daily). After two weeks of treatment, aphthous ulcers appeared on the lips and oral mucosa and topic acyclovir was prescribed. Two days later she developed fever, facial swelling and worsening of oral lesions with food refusal. After two days she was admitted to the paediatric department of a regional hospital. At this time she had bilateral facial swelling, with redness and warmth, exuberant gingivostomatitis with haemorrhagic plaques with fetid smell and some crusts, conjunctival injection and a generalised rash with maculopapular lesions on the limbs, palms and soles, back and face. She had two larger, non exudative, oedematous and erythematous cutaneous lesions - one on the neck; the other on the external face of the right arm $(15-20 \mathrm{~cm}$ of diameter). Blood tests only revealed increased C-reactive protein $(8.5 \mathrm{mg} / \mathrm{dL})$. Treatment with parenteral fluconazole, acyclovir and cefuroxime was started. As cutaneous lesions worsened and mucosal condition was exuberant she was transferred to our paediatric hospital.

The hypothesis of Stevens-Johnson syndrome (SJS) was considered and rufinamide was immediately stopped. Symptomatic and systemic treatment were instituted - amoxicillin with clavulanic acid and prednisolone ( $2 \mathrm{mg} / \mathrm{Kg} /$ day for 8 days followed by $1 \mathrm{mg} / \mathrm{Kg} /$ day for another 8 days). She was also on oral sucralfate, omeprazole and ocular chloramphenicol. Viral and bacterial infections were excluded by appropriate exams. A thorax X-ray was normal. During hospitalisation she was observed regularly by specialists in ophthalmology, stomatology and gastroenterology; no complications were found. She was observed by us on hospital day 6 . At that time, a clear improvement was noted. An evaluation using the Naranjo adverse drug reaction $(A D R)$ probability scale indicated that the causal relationship between rufinamide and the adverse reaction was probable (score of 6).

During hospital stay a favourable evolution was observed; she was afebrile since the second day, with progressive improvement of cutaneous lesions and aphthous condition and oral feeding was reintroduced. On the 13th day she returned to the regional hospital.

This is, to the best of our knowledge, the first case report of clinical SJS associated with rufinamide. A biopsy of lesional skin was not performed; we based our diagnosis on the clinical picture and on the score of the Naranjo ADR probability scale, because the availability of other diagnostic tests, in this case, is limited. ${ }^{5}$

Reliable skin test procedures in drug allergy are often lacking and test concentrations are unknown or poorly validated for most drugs. ${ }^{3}$ Intradermal tests (IDT) are usually sensitive but they must be performed with a parenteric form of the drug, which is not available in the case of rufinamide. Furthermore, there are safety concerns about the use of these tests in this type of reactions. ${ }^{3}$ The drug provocation test, which is the diagnostic gold standard in drug allergy, is contraindicated in potentially severe reactions like SJS. Lymphocyte transformation test (LTT), being an in vitro test, raises no safety concerns but can only be used to test drugs that exist in a soluble form, ${ }^{3}$ which is not the case with rufinamide. Drug patch tests (DPT) are safe and can be performed with any commercially available drug form, so they would be the only diagnostic test to use in this case. However, they have a low sensitivity and a negative result would not be reliable enough to allow a safe reintroduction of the drug. Furthermore, these tests would demand at least three visits to the hospital within a week, which would represent a considerable burden to the patient and her family, since they live in another city. On the other side, a positive result would not add crucial information for the diagnosis and management of this particular patient.

The Naranjo ADR probability scale is a validated scale that evaluates the probability of relationship between an adverse event and drug therapy based on a list of weighted questions, which examine factors such as the temporal association of drug administration and the event, alternative causes for the event, drug levels, dose-response relationships and previous patient experience with the medication. ${ }^{5}$

The diagnosis of SJS can be confirmed on histopathologic analysis of lesional skin. Histology reveals a subepidermal separation, and dermal infiltrates are located superficially and perivascularly. ${ }^{8,9}$ Early lesions show scattered necrotic keratinocytes in the epidermis with minimum T-cell infiltration; whereas late lesions show confluent full-thickness epidermal necrosis with subepidermal blisters. ${ }^{7}$ The most common immunohistochemistry findings are a mononuclear cell infiltrate composed mainly of activated $\mathrm{T}$ cells expressing DR antigens, CD69 activation markers, and the skin-homing receptor CLA in both CD4+ and CD8+ $T$ cells (with a general predominance of CD4+ cells). ${ }^{6}$ Given the fast improvement of her clinical state after the removal of rufinamide, it was considered that submitting this child to a skin biopsy was not necessary.

Concerning the therapeutic approach, there is continuing controversy regarding the use of systemic corticosteroids in the management of SJS. Several studies recommend its use to reduce morbidity and improve patient outcome. ${ }^{1,2}$ In the case of our patient there was an apparently favourable response. However, we still lack convincing evidenced-based proof for the beneficial effects of corticosteroids in the treatment of SJS patients. ${ }^{4}$ 
This is, to the best of our knowledge, the first case report of clinical SJS associated with rufinamide. All non-described severe reactions should be reported, regardless of the lack of diagnostic tests, in order to alert clinicians.

\section{References}

1. Koh MJ-A, Tay Y-K. An update on Stevens-Johnson syndrome and toxic epidermal necrolysis in children. Curr Opin Pediatrics. 2009;21:505-10.

2. Struck MF, Hilbert $P$, Mockenhaupt $M$, Reichelt B, Steen $M$. Severe cutaneous adverse reactions emergency approach to nonepidermolytic syndromes. Intensive Care Med. 2010;36:22-32.

3. Torres MJ, Mayorga C, Blanca M. Nonimmediate allergic reactions induced by drugs: pathogenesis and diagnostic tests. J Investig allergol Clin Immunol. 2009;19:80-90.

4. Wolf R, Davidovici B. Severe cutaneous adverse drug reactions: who should treat, where and how?: facts and controversies. Clin Dermatol. 2010;28:344-8.

5. Naranjo CA, Busto U, Sellers EM. A method for estimating the probability of adverse drug reactions. Clin Pharmacol Ther. 1981;30:239-45.

6. Torres MJ, Mayorga C, Fernandez TD, Cornejo-Garcia JA, Antunez $C$, Valenzuela $M$, et al. $T$ cell assessment in allergic drug reactions during the acute phase according to the time of occurrence. Int J Immunopathol Pharmacol. 2006;19: 119-30.

7. Mayorga C, R Pena R, Blanca-López N, López S, Martín E, Torres MJ. Monitoring the acute phase response in non-immediate allergic drug reactions. Curr Opin Allergy Clin Immunol. 2006;6:249-57.

8. Bastuji-Garin S, Rzany B, Stern RS, Shear NH, Naldi L, Roujeau JC. Clinical classification of cases of toxic epidermal necrolysis. Stevens-Johnson syndrome, and erythema multiforme. Arch Dermatol. 1993;129:92-6.

9. Rzany B, Hering O, Mockenhaupt M, Schröder W, Goerttler E, Ring J, et al. Histopathological and epidemiological characteristics of patients with erythema exudativum multiforme major, Stevens-Johnson syndrome and toxic epidermal necrolysis. $\mathrm{Br} \mathrm{J}$ Dermatol. 1996;135:6-11.

M. Chambel ${ }^{a, *}$, M.I. Mascarenhas ${ }^{b}$, J. Regalac ${ }^{c}$,

C. Gouveia ${ }^{c}$, S. Prates ${ }^{a}$

a Immunoallergy Department, Dona Estefânia Hospital, Lisbon Central Hospital, Rua Jacinta Marto, 1169-045 Lisbon, Portugal

b Immunoallergy Department, Santa Maria Hospital, Lisbon North Hospital, Lisbon, Portugal

c Paediatric Department, Dona Estefânia Hospital, Lisbon Central Hospital, Lisbon, Portugal

* Corresponding author.

E-mail address: chambel.marta@gmail.com (M. Chambel).

doi:10.1016/j.aller.2011.12.004 